\title{
Time-resolved TEM beyond fast detectors
}

\section{Flannigan, J. Chen, W. Curtis, D. Du, P. Engen, E. VandenBussche, Y. Zhang}

\author{
Department of Chemical Engineering and Materials Science, University of Minnesota, 421 Washington Avenue SE, Minneapolis, \\ Minnesota 55455, United States of America
}

flan0076@umn.edu

Timescales of dynamic processes in extended solids span many orders of magnitude owing to the large number of degrees of freedom. Additionally, scaling laws dictate that discrete temporal domains comprising the entire continuum consist of associated spatial domains within which specific dynamics are dominant. Ideally, one would be able to probe the entire spatiotemporal range on a single specimen spot with a single instrument. Modern TEMs are exceptionally versatile in this regard, providing access to spatial and energy ranges that span sub- $\AA$ to micrometres and sub-10 meV to $1,000 \mathrm{~s}$ of eV, respectively. However, timescales of the associated physical phenomena span 100 s of attoseconds $\left(10^{-18} \mathrm{~s}\right)$ to minutes and longer, a range that cannot be fully covered by even the fastest direct detectors. Indeed, dynamics faster than $\sim 0.1 \mathrm{~ms}$ are largely inaccessible to detector-based TEM approaches.

Here, an overview will be provided of ongoing efforts aimed at pushing TEM temporal resolutions well beyond the limits imposed by detectors and by peak dose rates. Emphasis will be placed on laser-driven nanosecond single-shot and fs stroboscopic modalities, currently the two most widely used approaches (Figure 1) [1-3]. Common hardware configurations based on modified commercial TEM platforms will be described, and current state-of-the-art performance specifications will be discussed. This will be followed by a brief survey of discoveries and advances that have been made with imaging, diffraction, and spectroscopy. Particular focus will be placed on experiments that have led to deeper understanding of materials and to new physics [4]. The talk will conclude with a brief look toward new emerging approaches and expanded applications, such as pulsed-beam damage mitigation [5,6].
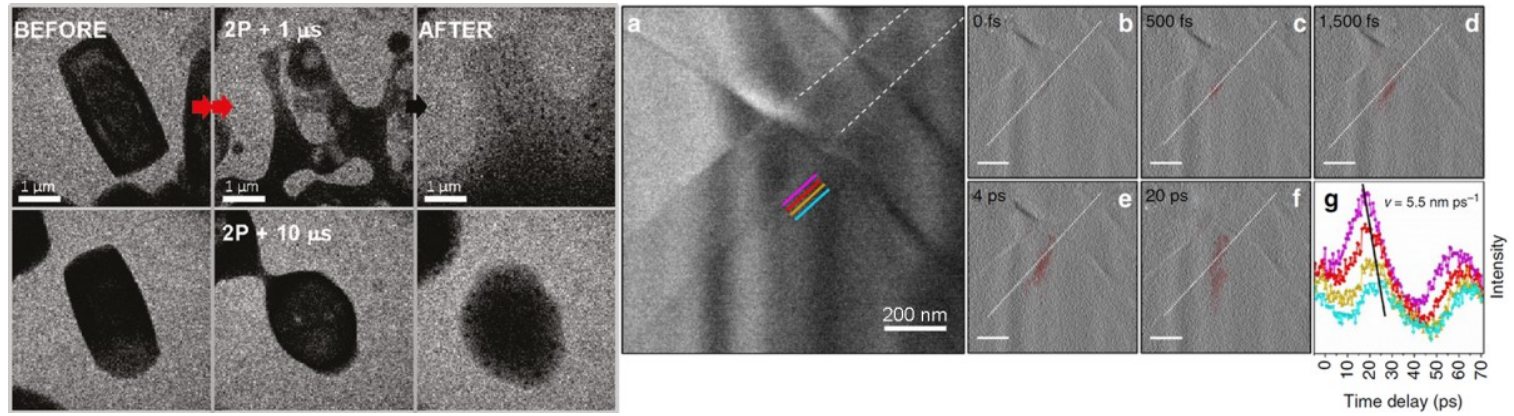

Figure 1. Nanosecond and fs TEM. Panels on the left are 10-ns single-shot images of a photoinduced redox reaction [1]. Panels on the right (a-g) are 300-fs stroboscopic images of photoexcited phonon launch from a crystal step edge [2].

[1] Park, S. T., Flannigan, D. J. \& Zewail, A. H. (2011). J. Am. Chem. Soc. 133, 1730.

[2] Cremons, D. R., Plemmons, D. A. \& Flannigan, D. J. (2016). Nat. Commun. 7, 11230.

[3] Plemmons, D. A., Suri, P. K. \& Flannigan, D. J. (2015). Chem. Mater. 27, 3178.

[4] Barwick, B., Flannigan, D. J. \& Zewail, A. H. (2009). Nature 462, 902.

[5] VandenBussche, E. J. \& Flannigan, D. J. (2019). Nano Lett. 19, 6687.

[6] VandenBussche, E. J., Clark, C. P., Holmes, R. J. \& Flannigan, D. J. (2020). ACS Omega 5, 31867.

\section{Keywords: 4D ultrafast electron microscopy; dynamic TEM; automation; synchronization; radiation damage}

This material is based on work supported by the U.S. Department of Energy, Office of Science, Office of Basic Energy Sciences under Award No. DE-SC0018204, by the U.S. Department of Energy through the UMN Centre for Quantum Materials under Grant No. DESC0016371, by the National Science Foundation under Grant No. DMR-1654318, partially by the National Science Foundation through the University of Minnesota MRSEC under Award Number DMR-2011401, and by the National Science Foundation Graduate Research Fellowship Program under Grant Nos. DGE-1348264 and DGE-1839286. Acknowledgement is made to the Donors of the American Chemical Society Petroleum Research Fund for partial support of this research.

Acta Cryst. (2021), A77, C420 